\title{
The scale effect of roughness in contact problems
}

\author{
S. Mezghani ${ }^{1}$, A. Jourani ${ }^{1}$ \& H. Zahouani ${ }^{2,3}$ \\ ${ }^{1}$ Laboratoire de Tribologie et Dynamique des Systèmes, \\ UMR CNRS, 5513, France \\ ${ }^{2}$ Ecole Nationale d'Ingénieurs de St Etienne, France \\ ${ }^{3}$ Ecole Centrale de Lyon, France
}

\begin{abstract}
In this paper we have used the Hölder exponent to characterise the scale of roughness and to study the scale effect of high spatial frequencies on elastic contact between solids. The mathematical approach shows that the Hölder exponent of roughness is a sophisticated tool for modelling realistic surface roughness at different scales of observation. The incidence of Hölder exponent on the prediction of pressure and displacement is studied in elastic contact between a smooth plane and rough surface.
\end{abstract}

Keywords: Holder exponent, scale effect, elastic contact.

\section{Introduction}

Surface topography plays an important role in a multitude of physical and tribological phenomena such as contact mechanics, friction, adhesion, wear, wettability, lubrication, etc. Surface topography causes discrete contact points, when two rough nominally flat surfaces are brought together, the real area of contact is the accumulation of the area of the individual contact points. For most metals at normal loads, this will be only a small percentage of the apparent contact area. Typical models of surface deformation are either elastic, plastic or mixed elastic-plastic, and can be represented schematically as a function of surface topography and material constants $f\left(\sigma_{\mathrm{h}}, \sigma_{\mathrm{s}}, \mathrm{R}_{\mathrm{c}}, \mathrm{E}, \mathrm{H}\right)$ with $\sigma_{\mathrm{h}}$ the root mean square of height, $\sigma_{s}$ the root mean square of summits, $R_{c}$ the mean radius of summit curvature, E and $\mathrm{H}$ are respectively the Young's modulus and the hardness of the solid which are the intrinsic parameters of the solid. On the other 
hand the principal statistical parameters of roughness, such as variance of height, slope and curvature are unfortunately not always independent of length scales. The definition of slope or summit of surface roughness systematically involves a high wave vector cut off due to the measurement process itself and not to the surface characteristics. Consequently, instruments with different resolutions and scan lengths yield different values of these statistical parameters for the same surface. Therefore it is very important to characterize rough surfaces by intrinsic parameters which are independent of the sampling length or area.

Hölder exponent which indicates the scale of perturbation of surface topography has been widely used in recent years [1-5], this is due in part to the observations that fractal geometry can reflect an intrinsic property of random phenomena and can be applied to characterize surface topography and contact mechanics. Majundar and Bhushan [5,7] explored these applications using the theory developed by Mandelbrot [6] concerning the cumulative distribution of islands on the earth's surface relief, which follows the power law $N \approx a^{-\frac{D}{2}}$, where $\mathrm{N}$ is the total number of islands whose area larger than $\mathrm{a}$, and $\mathrm{D}$ is the fractal dimension of its coast line $(0<\mathrm{D}<1)$.

In this work we have used the Hölder exponent to characterize the scale of roughness and to model the effect of roughness scale in contact mechanics.

In the second part we have studied, through experiments, the mechanism of asperity deformation using two numerical approaches based on geomorphologic characterization and fractal geometry using the concept developed by Mandelbrot.

\section{Elastic model of rough contact}

The majority of the methods used to determine the relationship between normal load and displacements, except finite elements methods, are based on the assumption of elastic solid, homogeneous and semi - infinite The basic assumptions necessary to use the continuous equations of elasticity:

The slopes of roughness are weak;

The contact area is small in front of other dimensions.

With zero loads, surfaces of the two bodies in the immediate neighbourhoods of the point of contact can be comparable with paraboloids of equations:

$$
\mathrm{z}_{1}=\mathrm{A}_{1} \mathrm{x}^{2}+\mathrm{A}_{2} \mathrm{xy}+\mathrm{A}_{3} \mathrm{y}^{2}, \quad \mathrm{z}_{2}=\mathrm{B}_{1} \mathrm{x}^{2}+\mathrm{B}_{2} \mathrm{xy}+\mathrm{B}_{3} \mathrm{y}^{2}
$$

Each surface is characterized at the point of contact by its principal radii of curvature $R_{1}$ and $R_{1}^{\prime}, R_{2}$ and $R_{2}^{\prime}$ is the angle which forms between them.

Constant $\mathrm{A}$ and $\mathrm{B}$ depend on the size of the principal curves of surfaces and the angle $\gamma$ with $A+B=\left(\frac{1}{R_{1}}+\frac{1}{R_{1}^{\prime}}+\frac{1}{R_{2}}+\frac{1}{R_{2}^{\prime}}\right)$.

By choosing an orientation of axes $\mathrm{X}$ and $\mathrm{Y}, \mathrm{X} 1$ and $\mathrm{Y} 1$, the terms in $\mathrm{xy}$ become negligible. The expressions of the equation (1) become: 


$$
z_{1}=\frac{1}{2 R_{1}^{\prime}} x_{1}^{2}+\frac{1}{2 R_{1}^{\prime \prime}} y_{1}^{2}, z_{2}=-\left(\frac{1}{2 R_{2}^{\prime}} x_{2}^{2}+\frac{1}{2 R_{2}^{\prime \prime}} y_{2}^{2}\right)
$$

The geometry of the contact between two unspecified solids is thus brought back to the problem of the contact between two paraboloids:

$$
h(x, y)=A x^{2}+B y^{2}=\frac{1}{2 R_{1}} x^{2}+\frac{1}{2 R_{2}} y^{2}
$$

The equations of linear elasticity make it possible to connect displacement $\mathrm{w}_{\mathrm{i}}(\mathrm{x}$, $y)$ of a $M(x$ point, y) of the surface of a semi elastic body infinite, with the pressure $p\left(x^{\prime}, y^{\prime}\right)$ in $\mathrm{Me}\left(x^{\prime}, y^{\prime}\right)$ pertaining to the surface of contact, by means of the following integral equation of Boussinesq:

$$
w_{i}(x, y)=\frac{1-v_{i}^{2}}{\pi E_{\text {equ }}} \iint_{A c} \frac{p\left(x^{\prime}, y^{\prime}\right) d x^{\prime} d y^{\prime}}{\sqrt{\left(x-x^{\prime}\right)^{2}+\left(y-y^{\prime}\right)^{2}}}
$$

with Ac the surface of contact

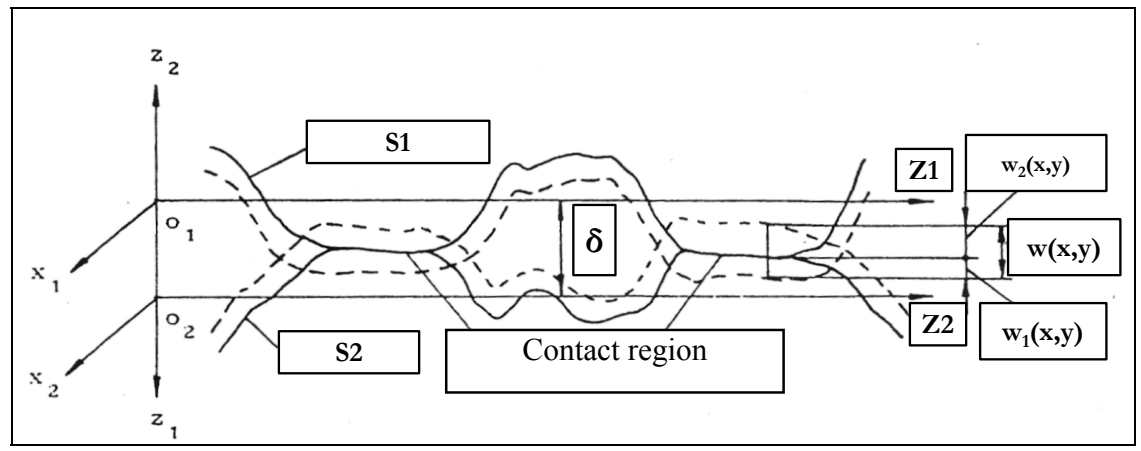

Figure 1: General diagram of the areas of contact between two solids.

So if one calls $w(x, y)$, the difference between the $M_{1}\left(x\right.$ point, $\left.y, z_{1}\right)$ of the surface of the solid $1(\mathrm{~S} 1)$ and the $\mathrm{M}_{2}\left(\mathrm{x}\right.$ point, $\left.\mathrm{y}, \mathrm{z}_{2}\right)$ of the surface of solid 2 (S2), the geometry enables us to write the following relation:

$$
w(x, y)=w_{1}(x, y)+w_{2}(x, y)=\delta-\frac{x^{2}}{2 R_{1}}-\frac{y^{2}}{2 R_{2}}
$$

where $\mathrm{w}_{1}(\mathrm{x}, \mathrm{y})$ and $\mathrm{w}_{2}(\mathrm{x}, \mathrm{y})$ are relative displacements on the two solids, $\mathrm{R}_{1}$ et $\mathrm{R}_{2}$ the principal radii of curvature of the two solids.

The determination of displacement in a point of surface due to the field of pressure is carried out by the superposition of the influence of all the efforts. Displacement at the point ij can then be written in the form: 


$$
w_{i j}=C_{i j k l} p_{k l}
$$

with $C_{i j k l}$ the matrix of the coefficients of influence.

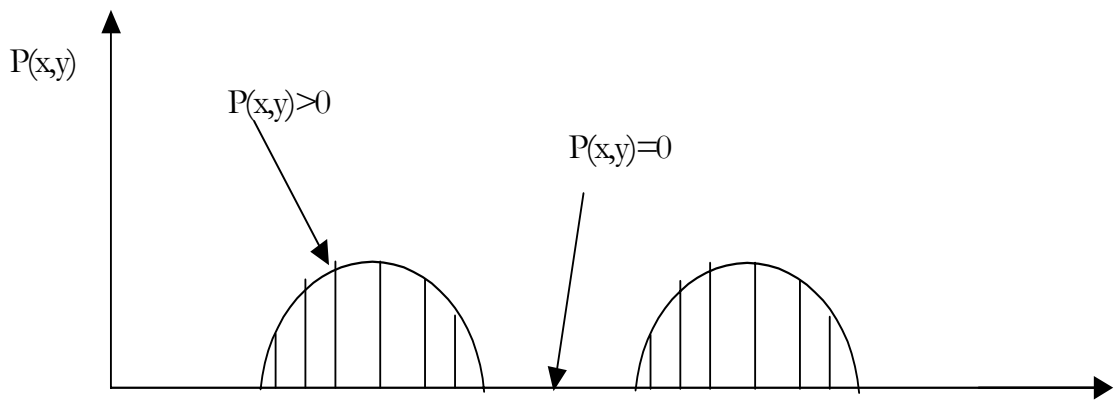

Figure 2: $\quad$ Pressures field at the contacts points between the two bodies.

The problem is thus to find pressures, such as $p_{i j}>0$, such as

$$
C_{i j k l} p_{k l}+h(x, y)-\delta=0
$$

If one consider the pressure as an input signal and displacement like a response of exit by the following relation to a dimension (Johnson 1985):

$$
w(x)=h(x) \otimes p(x)
$$

This equation is well defined in the space field in the form of a product of convolution: If the pressure applied is form

$$
p(x)=B_{0} \sin (2 \pi x / \lambda)
$$

The obtained displacement has the following expression (Johnson 1985)

$$
w(x)=\frac{\left(1-v^{2}\right) \lambda}{\pi E} B_{0} \sin (2 \pi x / \lambda)+c s t
$$

what gives like transfer function of the system in the field of the space frequencies:

$$
H(\omega)=\frac{2\left(1-v^{2}\right)}{E \omega}
$$

and in the field of the space frequencies one has like response

$$
W(\omega)=H(\omega) P(\omega)=\frac{2\left(1-v^{2}\right)}{E} \frac{P(\omega)}{\omega}
$$

In addition, by using the properties of the Fourier Transform one can write that the derivative of displacement can be written in Fourier domain as

$$
\dot{W}(\omega)=i \omega W(\omega)
$$

by replacing in the equation of the pressure one can write: 


$$
\begin{aligned}
& P(\omega)=\frac{E}{i 2\left(1-v^{2}\right)} \dot{W}(\omega) \\
& F(\omega)=\frac{E}{i 2\left(1-v^{2}\right)} W(\omega)
\end{aligned}
$$

These last relations are important characteristics of contact problems. They imply that the contact pressure and slope of displacement as well as the load and displacement are two pairs of parameters which have similar frequency composition.

The passage in the spectral field makes it possible to solve this last equation, while subjecting the functions a two-dimensional transform of Fourier. What makes it possible to write a relation between the spectrum of the pressures and the spectrum of displacements as $[1,2,3]$ :

$$
W\left(v_{x}, v_{y}\right)=\frac{2\left(1-v^{2}\right)}{E} \frac{P\left(v_{x}, v_{y}\right)}{v}
$$

where $v=\sqrt{v_{x}^{2}+v_{y}^{2}}$ represent a space frequency.

The determination of the field of pressure $\mathrm{p}(\mathrm{x}, \mathrm{y})$ is determined by opposite transform of Fourier for each level of bringing together $\delta$ :

$$
p_{\delta}(x, y)=\mathrm{TF}^{-1}\left[\frac{E}{2\left(1-v^{2}\right)} v W\left(v_{x}, v_{y}\right)\right]
$$

The spectrum of pressure is thus spread out between the low and high frequencies:

$$
v_{x}=\frac{1}{L x}, v_{y}=\frac{1}{L y} \mathrm{~mm}^{-1}, v_{h x}=\frac{1}{2 \Delta x}, v_{h y}=\frac{1}{2 \Delta y} \mathrm{~mm}^{-1}
$$

with $L x, L y$, the size of the surface, $\Delta x, \Delta y$ the sampling steps in the $\mathrm{x}$ and $\mathrm{y}$ directions, which initially define rough surface before contact.

For normal macroscopic imposed load F, one numerically brings closer two surfaces and to solve the equation for each level of bringing together $\delta$. For each normal position $\delta$ of the rigid plan, one determines contact pressures $\mathrm{p}(\mathrm{x}, \mathrm{y})$ by using the direct and inverse Fourier transform of the contact equations. Convergence is reached when the imposed effort is equal to the sum of the local efforts calculated for each iteration:

$$
F=\sum_{i, j} p_{i j}(x, y) \Delta x \Delta y
$$




\section{Hölder exponent of rough surfaces}

As the details of roughness: $Z(x)$ depends on the length scale, we assume each realization of $\mathrm{Z}(\mathrm{x})$ to be a continuous, but non-differentiable function. It means that the presence of any small roughness elements may prevent us from reaching a satisfactory limit of

$$
\frac{(Z(x+\Delta)-Z(x))}{\Delta} \text { as } \Delta \rightarrow 0
$$

with $\Delta$ the sampling step of roughness measurement

A simple way to obtain this behaviour for a function $\mathrm{Z}(\mathrm{x})$ is to assume that the increment of $\mathrm{Z}(\mathrm{x})$ is related to $\Delta$ by the self-affinity relation:

$$
\begin{gathered}
|Z(x+\Delta)-Z(x)| \propto \Delta^{H}, 0<H<1 \quad \Delta \rightarrow 0 \\
\lim _{\Delta \rightarrow 0} \Delta^{H-1}, \text { only exists if } \mathrm{H}=1 .
\end{gathered}
$$

The derivative of $\mathrm{Z}$, which is proportional to the limit, for $0<\mathrm{H}<1$, this derivative is infinite, although the function remains continuous, and for $\mathrm{H}=0$ the function itself becomes discontinuous. So by varying it from 0 to 1 , parameter $\mathrm{H}$ characterizes the transition from a non continuous to a differentiable function, the range $0<\mathrm{H}<1$ corresponding to non differentiable functions which become smoother as $\mathrm{H}$ increases. Thus $\mathrm{H}$ can be considered as an indicator of roughness. Since we are concerned with functions which can be considered as representing random processes, we state the self-affinity relation in terms of variance of the increments of Z:

$$
\left\langle(Z(x+\Delta)-Z(x))^{2}\right\rangle \propto \Delta^{2 H} \quad 0<\mathrm{H}<1, \Delta \rightarrow 0
$$

This relation can be generalized to dimensions $\mathrm{E}>1$ in the following way

$$
\left\langle(Z(x+\Delta)-Z(x))^{2}\right\rangle \propto\|\Delta\|^{2 H}, 0<\mathrm{H}<1, \Delta \rightarrow 0
$$

where $\mathrm{x}$ represents a point in E-dimensional Euclidean space $\mathrm{R}^{\mathrm{E}}$ and $\|$ || stands for the usual norm in this space. This is not a new condition; it is proven for all $\|\Delta\|$ values by the fractional Brownian motions.

\section{The spectral exponent}

From the previous relation it appears that the functions

$$
\mathrm{Z}(\mathrm{x}+\Delta)-\mathrm{Z}(\mathrm{x}) \text { and } \frac{(Z(x+s \Delta)-Z(x))}{s^{H}}
$$

Considered as functions of $\Delta$, have the same law of probability. If we assume that $Z(0)=0$, then function $Z(x)$ verifies the statistical self- affinity relation

$$
Z(s x)=s^{H}(Z(x)
$$


It means that the two functions $\mathrm{Z}(\mathrm{x})$ and $Y(x)=\frac{Z(s x)}{s^{H}}$ are statistically indistinguishable.

Voss (1985) showed that the fractal dimension is related to parameter $\mathrm{H}$ by the relation $\mathrm{D}=\mathrm{E}+1-\mathrm{H}$ where $\mathrm{E}$ is the dimension of the Euclidean space $\mathrm{R}^{\mathrm{E}}$. These functions, which correspond to a non-stationary random process with stationary increments, present many other interesting properties. For instance their power spectrum $S(\omega)$ presents a well-defined decrease following the power law

$$
S(\omega)=\frac{1}{\omega^{\beta}}=\frac{1}{\omega^{2 H+E}}, \quad \beta=2 H+E
$$

The power spectrum of roughness verifies another self-affinity relation

$$
S(k \omega)=\frac{1}{k^{2 H+E}} S(\omega)
$$

If one makes the analogy with the relation between the spectrum of pressure and the spectrum of the slopes of displacements which vary in the same space frequency band, one can notice that the law of power spectrum of pressure depends on the Hölder exponent as following

$$
\begin{gathered}
P(\omega)=\frac{E}{i 2\left(1-v^{2}\right)} \dot{W}(\omega) \\
P(k \omega)=\frac{1}{k^{2 H+E}} P(\omega)
\end{gathered}
$$

This relation, shows well the effect of the exhibitor of Hölder on the spectrum of pressure, and consequently on the local singularities of pressures and displacements which will induce local plasticity.

\section{The effect of the Hölder exponent of roughness on elastic contact}

In order to study the influence of the Hölder exponent of roughness on the parameters of elastic contact: pressure, displacement and real contact area, we propose to generate three rough surfaces parameterised in roughness by their Hölder exponent. Each of those surfaces is the sum of a periodic surface (sinusoidal) and a random rough surface generated by the random displacement model [14-15] with different roughness Hölder exponent $\mathrm{H}=0.1, \mathrm{H}=0.5, \mathrm{H}=0.8$ (figure 3). Those surfaces obtained (figure $4\{\mathrm{a} 1, \mathrm{a} 2, \mathrm{a} 3\}$ ) were put in contact under a pressure of $400 \mathrm{MPa}$ with a rigid plan.

Pressure fields are calculated for each of these three rough surfaces as using the model of the elastic contact (figure 4). We notice that the pressure field gotten is as much more discontinuous than the surface is rougher. 


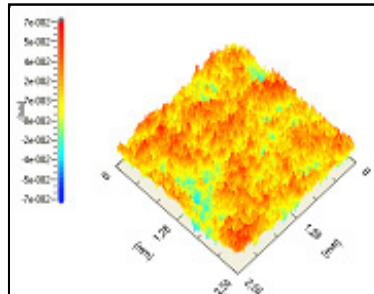

(a)

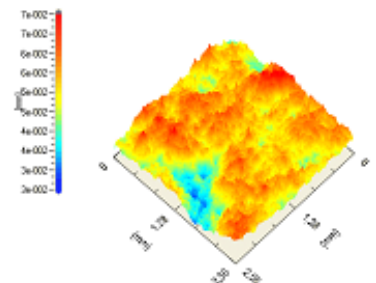

(b)

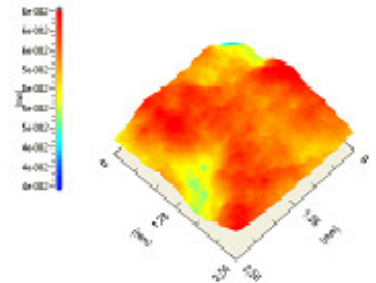

(c)

Figure 3: Three examples of simulation by the algorithm of the midpoint method with random successive additions (a) $\mathrm{H}=0.1$ (b) $\mathrm{H}=0.5$ (c) $\mathrm{H}=0.8$.

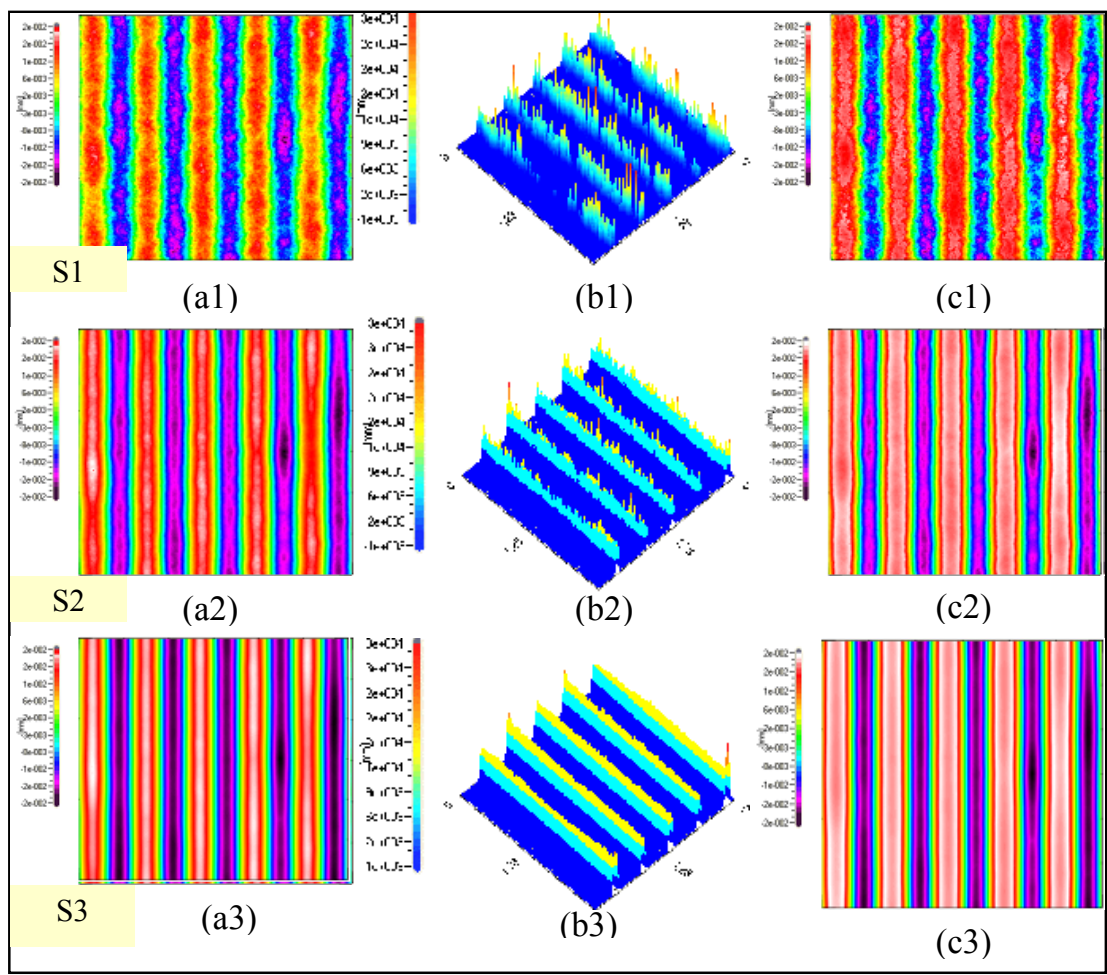

Figure 4: Roughness surfaces: (a1) $\mathrm{H}=0.1$, (a2) $\mathrm{H}=0.5$, (a3) $\mathrm{H}=0.8$; Pressure field: (b1) $\mathrm{H}=0.1$, (b2) $\mathrm{H}=0.5$, (b3) $\mathrm{H}=0.8$; deformed surfaces: (c1) $\mathrm{H}=0.1$, (c2) $\mathrm{H}=0.5$, (c3) $\mathrm{H}=0.8$; $\{$ Applied pressure $400 \mathrm{MPa}$.

The following table (Table 1) regroups the whole of contact parameters below (Bearing surface area and mean contact pressure) of the three different rough surfaces. 
Table 1: $\quad$ Contact parameters for various roughness surfaces.

\begin{tabular}{|c|c|c|}
\hline Surface roughness & Bearing area (\%) & Mean Pressure (GPa) \\
\hline $\mathrm{S} 1(\mathrm{H}=0.1)$ & 11.66 & 3.40 \\
\hline $\mathrm{S} 2(\mathrm{H}=0.5)$ & 27.78 & 1.41 \\
\hline $\mathrm{S} 3(\mathrm{H}=0.8)$ & 28.60 & 1.38 \\
\hline
\end{tabular}

These results (Table 1) show that the roughest surface (S1) which has the lower Holder exponent is the more resistant in contact. Indeed, it presents the lowest bearing area surface $(11.66 \%)$ and the most important mean pressure $(3.40 \mathrm{GPa})$. This proves the narrow relation between punctual pressure field's singularity and the Holder exponent indicator of roughness.

\section{Conclusion}

The incidence of the Hölder exponent of roughness is important in contact mechanics, wettability and lubrication of rough material, where the knowledge of the surface bearing area, the developed area or void volume is directly related to the scale of observation. In contact mechanics there are two incidences. The first is related to the size of the spot area and surface bearing area, the second is connected to the evolution of the void and material volume during contact as a function of observation scale. The mathematical modelling study shows that the Hölder exponent of roughness is a sophisticated tool for modelling realistic surface roughness at different scale of observation. It can be used as the input data in contact mechanic modelling. This scale parameter can be used as an indicator of the real length, area and volume of rough material, independently of the scale of observation. This scale parameter is very promising to simulate the behaviour of rough morphology in static contact.

\section{References}

[1] Johnson K.L., N - J Mech. Phys. Solids - 1970.

[2] Ju Y., Farris T.N., Spectral analysis of two dimensional contact problems, Transactions of the ASME, Vol 118, pp 320-329, 1996.

[3] Liu Sh., Wang Q., Liu G., A versatile method of discrete convolution and FFT for contact analyses. Wear 243 pp. 101-111, 2000.

[4] Gagnepin J.J and Roques-Carmes C., Fractal approach to twodimensional and three-dimensional surface roughness, Wear, Vol 109, pp.119-114, 1986.

[5] Majunder A. and Bhushan B., Role of fractal geometry in roughness characterisation and contact mechanics of surfaces, ASME J. Tribology, Vol. 112, pp.205-216, 1990.

[6] Majunder A. and Tien C.L, Fractal characterisation and simulation of rough surfaces, Wear, Vol 136, pp. pp. 313-327, 1990.

[7] Majunder A. and Bhushan B., Fractal model of elastic-plastic contact between rough surfaces, J. Tribology, Vol 13, pp.1-11. 1991. 
[8] Lopez J., Hansali G., Zahouani H., Lebossé J.C., Mathia T., 3D Fractalbased characterisation for engineered surface topography, Int.J.Mach.Tools Manufact, Vol 35, pp.211-217, 1995.

[9] Mandelbrot B., Stochastic models for the Earth's relief, the shape and the fractal dimension of coastlines, and the number-area rule for islands, Proceedings of the National Academy of Science, Vol 72, pp.3825-3838, 1975.

[10] Richardson L.F., The problem of contiguity: An appendix of statistics of deadly quarrels, General Systems Yearbook 6, pp.687-689, 1961.

[11] Mandelbrot B., How long is the coast of Britain - Statistical self-similarity and fractional dimension, Science, Vol 155, pp.636-638, 1967.

[12] Brown S.R. and Scholz C.H., Broad bandwidth study of the topography of natural rock surfaces, J. Geophys. Res., 90, pp. 12575-12582. , 1985.

[13] Felder J., Fractals, Plenum Press, New York, 1988.

[14] Mandelbrot B. and Van Ness J.W, Fractional Brownian motions, fractional noises, and applications, SIAM Review 10, 422-437, 1968.

[15] Russ John C., Fractal surfaces, Plenum Press, New York, 1944.

[16] Saupe D. and Peitgen H.O., The science of fractal images. SpringerVerlag pp.82-91, 1988.

[17] Zahouani H., Vargiolu R., Fractal models of surface topography and contact mechanics, Vol28, N 4-8, pp 517 - 534, 1998. 